\title{
Implicações do diagnóstico precoce da deficiência de glicose-6-fosfato desidrogenase (G6PD) no prognóstico neurológico de neonatos
}

\author{
Implications of early diagnosis of glucose-6-phosphate dehydrogenase (G6PD) deficiency \\ in the neurological prognosis of neonates
}

\begin{abstract}
Implicaciones del diagnóstico precoz de la deficiencia de glucosa-6-fosfato deshidrogenasa (G6PD) en el pronóstico neurológico de los recién nacidos
\end{abstract}

Bianca Carollyne Martins Pinto ${ }^{1 *}$, Amanda Moreira Gonçalves ${ }^{1}$, Amanda Dantas Sabbi ${ }^{2}$, Pedro Antonio Rodrigues Dias ${ }^{3}$, Cecília Braga Balsamão ${ }^{4}$, Rafael Oliveira Halfeld ${ }^{5}$, Sofia Silva e Souza ${ }^{6}$, Zhandra Gramigna Giampietro ${ }^{7}$, Rayane Cristina Batista Rodrigues ${ }^{3}$.

\section{RESUMO}

Objetivo: Compreender a importância de realizar diagnósticos ágeis de deficiência de Glicose-6-Fosfato Desidrogenase (G6PD) a fim de evitar sequelas neurológicas graves em lactentes. Revisão bibliográfica: Foram selecionados estudos que abordassem população de 0 a 23 meses de idade e complicações neurológicas decorrente de hiperbilirrubinemia patológica. Constatou-se que essa doença hemolítica possui incidência universal, embora não seja rastreada difusamente. Por ser assintomática e nem sempre estar disponível a triagem neonatal, há brechas para evoluir para sua mais grave complicação, kernicterus. Dessa forma, é preconizado a instituição de tratamento enquanto a encefalopatia aguda é reversível, o qual consiste principalmente em fototerapia e transfusão sanguínea. Especialmente em lugares onde não se realiza a busca ativa de casos, o tratamento deve estar aliado à conscientização dos pais, que devem estar vigilantes quanto à icterícia e realizar busca rápida de assistência médica, uma vez que o momento de maior vulnerabilidade é após a alta hospitalar do recém-nascido. Considerações finais: A deficiência de G6PD é relevante e pode colaborar para evoluções graves do quadro de hiperbilirrubinemia. Há limitações na abordagem dos neonatos acometidos, com escassez de estudos na área e reflexos da falta de investimentos no prognóstico destes.

Palavras-Chave: Deficiência de Glucose-6-fosfato desidrogenase, Icterícia neonatal, Hiperbilirrubinemia neonatal, Kernicterus.

\section{ABSTRACT}

Objective: Understand the importance of making prompt diagnoses of Glucose-6-Phosphate Dehydrogenase (G6PD) deficiency in order to avoid severe neurological sequels in infants. Bibliographic review: Studies were selected that include a population from 0 to 23 months of age and neurological complications resulting from pathological hyperbilirubinemia. It was found that this hemolytic disease has a universal incidence, although it is not diffusely tracked. Because this deficiency in G6PD does not manifest symptoms and neonatal screening is not always available, there are loopholes to progress to its most serious complication, kernicterus. Thus, the institution of treatment is recommended while the acute encephalopathy is reversible, which consists mainly of phototherapy and blood transfusion. Especially in places where the active search for cases is not carried out, it is understood that the treatment must be coupled with the awareness of the parents, who must be vigilant about jaundice and carry out a quick search for medical assistance, since the moment of greatest vulnerability is after the newborn's hospital discharge. Final considerations: G6PD deficiency is relevant and can contribute to serious developments in hyperbilirubinemia. There are limitations in the approach of affected neonates, with a shortage of studies in the area and reflections of the lack of investments in their prognosis.

Keywords: Glucose-6-phosphate dehydrogenase deficiency, Jaundice neonatal, Hyperbilirubinemia neonatal, Kernicterus.

\footnotetext{
${ }_{1}$ Pontifícia Universidade Católica de Minas Gerais (PUC Minas), Betim - MG.

*E-mail: biancacmartinsp@gmail.com

2 Centro Universitário de Várzea Grande (UNIVAG), Várzea Grande - MT.

${ }^{3}$ Centro Universitário de Caratinga (UNEC), Caratinga - MG.

${ }^{4}$ Centro Universitário de Belo Horizonte (UNIBH), Belo Horizonte - MG.

${ }^{5}$ Universidade do Vale do Sapucaí (UNIVAS), Pouso Alegre - MG.

${ }^{6}$ Faculdade de Minas (FAMINAS), Belo Horizonte - MG.

7 Universidade Federal do Amapá (UNIFAP), Macapá - AP.
} 


\section{RESUMEN}

Objetivo: Comprender la importancia de realizar diagnósticos rápidos de la deficiencia de glucosa-6-fosfato deshidrogenasa (G6PD) para evitar secuelas neurológicas graves en los lactantes. Revisión bibliográfica: Se seleccionaron estudios que abordaban una población de 0 a 23 meses de edad y las complicaciones neurológicas resultantes de la hiperbilirrubinemia patológica. Se descubrió que esta enfermedad hemolítica tiene una incidencia universal, aunque no se realiza un seguimiento difuso. Debido a que el cribado neonatal es asintomático y el cribado neonatal no siempre está disponible, hay lagunas para evolucionar a su complicación más grave, kernicterus. Por lo tanto, se recomienda la institución del tratamiento, mientras que la encefalopatía aguda es reversible, que consiste principalmente en fototerapia y transfusión de sangre. Especialmente en lugares donde no hay búsqueda activa de casos, se entiende que el tratamiento debe ir acompañado de la conciencia de los padres, quienes deben estar atentos a la ictericia y realizar una búsqueda rápida de asistencia médica, desde el momento de mayor La vulnerabilidad es después del alta hospitalaria del recién nacido. Consideraciones finales: La deficiencia de G6PD es relevante y puede contribuir a desarrollos graves en la hiperbilirrubinemia. Existen limitaciones en el enfoque de los recién nacidos afectados, con escasez de estudios en el área y reflejos de la falta de inversiones en su pronóstico.

Palabras clave: Deficiencia de glucosa-6-fosfato deshidrogenasa, Ictericia Neonatal, Hiperbilirrubinemia neonatal, Kernicterus.

\section{INTRODUÇÃO}

A Glucose 6-fosfato desidrogenase (G6PD) é uma enzima encontrada em todas as células do corpo. Catalisadora da primeira etapa na via da pentose fosfato, com a função de proteger as células contra danos oxidativos, a G6PD converte a coenzima Nicotinamida Adenina Dinucleotídeo Fosfato (NADP) em sua forma reduzida Nicotinamida Adenina Dinucleotídeo reduzida (NADPH), sendo, praticamente, toda fonte geradora de NADPH, que tem como função combater os efeitos prejudiciais das espécies reativas de oxigênio (IGLESSIAS MAC, et al., 2010).

Essa via desempenha papel fundamental na proteção dos glóbulos vermelhos frente aos processos oxidativos, que são responsáveis pela redução da vida média das hemácias. Nos eritrócitos deficientes de G6PD, há um baixo potencial redutor que interfere diretamente na capacidade metabólica oxidativa do organismo, tornando-se vulnerável a hemólise (IGLESSIAS MAC, et al., 2010; FRANK JE, 2005).

A deficiência de G6PD é de longe a eritroenzimopatia mais prevalente, afetando mais de 400 milhões de pessoas em todo o mundo, principalmente na África, países mediterrâneos e sudoeste da Ásia. A maioria dos indivíduos deficientes são assintomáticos e podem exibir crises hemolíticas de intensidade variável e icterícia neonatal, após ingestão de certas drogas oxidantes, processos infecciosos e oxidativos (IGLESSIAS MAC, et al., 2010; LEITE AA, 2010). No Brasil, a estimativa é de 6 milhões de afetados, com aproximadamente $1 \%$ dos neonatos apresentando episódios de icterícia neonatal, ou seja, 1,9 milhões de recém-nascidos sujeitos ao risco de desenvolver um quadro sintomático mais grave (NKHOMA ET, et al., 2009; LEITE AA, 2010).

Bebês com deficiência de G6PD são significativamente mais propensos a desenvolver hiperbilirrubinemia, apresentando taxas 4 vezes maiores em comparação com indivíduos normais, além de possuírem 3 vezes mais chances de receber fototerapia (CUNNINGHAM AD, et al., 2016). No período neonatal, a complicação mais severa que a deficiência de G6PD pode causar é a encefalopatia bilirrubínica crônica (EBC) ou Kernicterus, caracterizado por letargia, diminuição da alimentação, choro agudo, febre, convulsões e que pode agravar para uma paralisia cerebral do tipo atetóide ou ao óbito (LEITE AA, 2010).

É difícil estimar a incidência de Kernicterus devido ao atraso no diagnóstico, subnotificação ou falta de notificação nos países do terceiro mundo. A incidência de Kernicterus nos países em desenvolvimento é maior devido a uma variedade de fatores, como infraestrutura deficiente, falta de recursos, diferenças genéticas, prematuridade, baixo peso ao nascer, parto domiciliar, subnutrição e sepse (FARIA DC, et al., 2016).

Diante desse contexto, o presente estudo tem como orientação a seguinte pergunta: "Como o diagnóstico precoce da deficiência de G6PD pode favorecer o prognóstico do quadro de Kernicterus por hiperbilirrubinemia patológica em recém-nascidos?". Acredita-se que, a partir do diagnóstico precoce dessa 
deficiência, medidas profiláticas para hiperbilirrubinemia possam ser definidas com o intuito de impedir o desenvolvimento da encefalopatia bilirrubínica ou amenizar danos futuros decorrentes dela. Assim, define-se como objetivo dessa pesquisa analisar como o diagnóstico precoce da deficiência de G6PD relaciona-se com o melhor prognóstico decorrente da hiperbilirrubinemia patológica.

\section{REVISÃO BIBLIOGRÁFICA}

\section{Fisiopatologia}

A G6PD faz parte da via das pentoses. Por meio dessa via, as enzimas G6PD e 6-fosfogliconato desidrogenase (6PGD) reduzem a coenzima NADP à NADPH, que então passa a atuar como coenzima doadora de hidrogênio em sínteses redutoras. O contrabalanceamento do estresse oxidativo na hemoglobina pode levar à hemólise devido ao monóxido de carbono existente no glóbulo vermelho, podendo culminar em um quadro ictérico.

Esse processo hemolítico é caracterizado por: bilirrubina não conjugada, desidrogenase lática e reticulócitos, os quais vão estar aumentados. Segundo a Academia Americana de Pediatria, a hemólise é a maior causadora de hiperbilirrubinemia severa. Dessa forma, a G6PD atua protegendo o grupo sulfidrilas da hemoglobina de uma desnaturação oxidativa e evita a formação de corpos de Heinz, que consistem na precipitação de cadeias de globina que se liberam do grupo heme quando a hemoglobina é oxidada (FARIA DC, 2016; KAPLAN M, et al., 2004; KAPLAN M e HAMMERMAN C, 2002; LEITE AA, 2010; OMS, 1989).

O fígado do recém-nascido é incapaz de conjugar adequadamente toda a bilirrubina indireta que provém da hemólise, culminando em um acúmulo de bilirrubina indireta sérica, resultando assim na icterícia. A icterícia pode ser dividida em dois grupos: a fisiológica e a patológica.

No primeiro grupo, a icterícia ocorre após 24 horas de nascimento do bebê, devido a adaptação do neonato ao metabolismo de bilirrubina sérica, com valor de até $12 \mathrm{mg} / \mathrm{dl}$. No segundo grupo, entre outras causas existentes, a icterícia pode ocorrer por deficiência da enzima G6PD, uma doença de causa genética, recessiva e ligada ao cromossomo $X$, o que explica sua maior frequência no sexo masculino. A deficiência dessa enzima é definida por sua atividade menor que $7,0 \mathrm{IU} / \mathrm{gHB}$ e faz parte do grupo das doenças hemolíticas de causas enzimáticas que resultam em uma icterícia patológica, de surgimento precoce, nas primeiras horas de vida (OSHIRO M, et al., 2014; WATCHKO J, 2017).

Embora o mecanismo da neurotoxicidade induzida por bilirrubina não seja completamente compreendido, é claro que a deficiência de G6PD contribui para o Kernicterus por pelo menos dois mecanismos: inicialmente, através da elevação induzida por hemólise nos níveis de bilirrubina indireta e subsequente acúmulo de bilirrubina no cérebro e segundo, pela capacidade tampão reduzida contra espécie reativa de oxigênio (ERO) induzida por bilirrubina.

Se a hiperbilirrubinemia grave não for tratada, a bilirrubina pode atravessar a barreira hematoencefálica, levando à encefalopatia aguda da bilirrubina, que é reversível se identificada e tratada precocemente; se crônico, pode resultar em Kernicterus, caracterizado por letargia, diminuição da alimentação, choro agudo, febre, convulsões e até morte (FARIA DC, et al., 2016).

\section{Repercussões}

Os estudos demonstram que a maior parte dos pacientes portadores da deficiência de G6PD são assintomáticos. Foi observado que mesmo uma grande redução na atividade da G6PD tem pouco efeito clínico em circunstâncias comuns, mas pode se tornar dramaticamente aparente na presença de estresse oxidativo, como exposição do recém-nascido a certos medicamentos, alimentos, produtos químicos, infecções ou hipóxia. Nesses casos, pode se observar um quadro de anemia hemolítica normocítica e normocrômica, com reticulocitose evidente quatro a cinco dias após o início do episódio (WHO WG, 1989; ISA HM, et al., 2017).

Nos eritrócitos deficientes de G6PD, a diminuição da redução do NADP em NADPH leva a um baixo potencial redutor que interfere na capacidade metabólica oxidativa do organismo, ficando vulnerável a hemólise por não conseguir proteger os grupos sulfidrilas da hemoglobina com formação de corpos de Heinz, 
com oxidação da membrana do glóbulo, podendo levar a crises hemolíticas de intensidade variável e icterícia neonatal, após ingestão de certas drogas oxidantes, processos infecciosos e oxidativos. A consequência mais grave da deficiência de G6PD é uma acentuada icterícia neonatal, que pode levar a kernicterus (LEITE AA, 2010).

Laboratorialmente traduz-se por um aumento dos marcadores de hemólise: bilirrubina não conjugada, desidrogenase láctica e reticulócitos. Em relação a bilirrubina, o aumento da produção de bilirrubina juntamente com sua excreção ineficiente se traduzem em uma hiperbilirrubinemia, se apresenta aos exames de recém-nascidos do sexo masculino classificados como deficientes valores de $1,7 \mathrm{Ul} / \mathrm{gHb}$, enquanto no sexo feminino são considerados deficientes valores de 3,58 Ul/gHb (CUNNINGHAM AD, et al., 2016; FARIA DC, et al, 2016; OSHIRO M, et al., 2014).

A hiperbilirrubinemia evolui para o principal sintoma relatado nos estudos, a icterícia neonatal, icterícia essa que tem como evolução mais severa o Kernicterus. O quadro clínico dos indivíduos portadores de kernicterus, pode compor-se de distúrbio do movimento distônico ou atóide, distúrbio do processamento auditivo associado à perda auditiva, comprometimento ocular motor do olhar para cima, displasia do esmalte dos dentes e hipotonia e ataxia devido ao envolvimento cerebelar (KAPLAN M, et al., 2011).

Kernicterus é uma sequela clínica mais crônica e permanente de toxicidade da bilirrubina em neonatos que sobrevivem à Encefalopatia Crônica por Bilirrubina. Evolui lentamente durante vários anos nas crianças afetadas. Na fase inicial, que ocorre no $1^{\circ}$ ano de vida, geralmente apresenta hipotonia, hiperreflexia, persistência de tonicidade reflexo do pescoço e marcos atrasados. Após o $1^{\circ}$ ano, a manifestação é mais variável, com uma série de sintomas incluindo anormalidades auditivas, visuais e dentárias, e distúrbios extrapiramidais (USMAN F, et al., 2018).

\section{Diagnóstico precoce}

O diagnóstico precoce da deficiência da enzima G6PD é a única forma de prevenção do kernicterus, fato que demanda um programa de rastreio universal dos neonatos ainda não existente. No entanto, a atividade da enzima G6PD só é testada quando o nível de icterícia é clinicamente detectável. A idade média de pico dessa icterícia nos recém-nascidos é cerca de 5 dias, período em que a maioria das crianças já se encontra de alta hospitalar, recebida, usualmente, após 48 horas pós parto. No momento da alta, grande parte dessas crianças apresentam-se saudáveis, porém é necessário atentar-se aos níveis de bilirrubina sérica, que podem se elevar de forma acelerada e provocar significativos danos neurológicos (NAIR PAK e AL KHUSAIBY SM, 2003).

A Organização Mundial da Saúde recomenda a triagem neonatal para deficiência de G6PD nos países que apresentam prevalência entre 3-5\% na população masculina, porém, as recomendações de rastreamento ainda variam muito de acordo com os sistemas de saúde público de cada país. Em relação ao Brasil, sabese que existe um programa de rastreio em neonatos disponibilizado pelo Sistema Único de Saúde para diversas patologias, o Teste do Pezinho - ou Teste de Guthrie -, o qual busca identificar a presença de seis doenças congênitas. No entanto, o teste é realizado entre o $3^{\circ}$ e $5^{\circ}$ dia de vida do bebê, o que se contrapõe à necessidade do recém-nascido receber o diagnóstico da deficiência de G6PD antes da alta hospitalar, motivo esse que fez com que a inclusão do rastreio para a enzimopatia no teste fosse recusada (BRASIL, 2018).

A preocupação maior está voltada aos locais que não possuem recursos suficientes para implementar um programa de rastreio. Com isso, no intuito de manter o diagnóstico antecedendo os sintomas da enzimopatia, propôs-se uma ferramenta clínica para reconhecer os sinais de perigo da encefalopatia aguda, quando a intervenção apropriada possui maior chance de limitar ou reverter a neurotoxicidade.

Essa ferramenta baseia-se no algoritmo de pontuação da disfunção neurológica induzida por bilirrubina (BIND), um sistema simples que mostra que até mesmo locais desprovidos de recursos podem dispor de uma triagem efetiva. Esse algoritmo atribui uma pontuação de 1 a 3 para indicar anormalidades leves, moderadas ou graves, respectivamente, no estado mental, tônus muscular e choro da criança. A partir disso, pontuações mais altas indicam sinais de piora da neurotoxicidade associada com hiperbilirrubinemia. (RADMACHER PG, et al., 2015). 
Tratando-se dos métodos diagnósticos utilizados, o teste de mancha fluorescente (FTS, fluorescent spot test em inglês) é amplamente utilizado para triagem qualitativa da deficiência de G6PD e utiliza manchas de sangue, sendo acessível e produzindo resultados em poucos minutos. A deficiência de G6PD foi definida como uma intensidade relativa de fluorescência inferior a 30\%. Como é um teste rápido e econômico, é recomendado pelo Comitê Internacional de Normalização em Hematologia (ICSH, Committee for Standardization in Hematology em inglês). Porém, possui limitações por exigir visualizações ultravioleta e habilidades específicas para interpretação (PENGBOON P, et al., 2019; WENG Y, et al., 2003).

O método de referência para detecção da enzimopatia é baseado em um ensaio enzimático quantitativo. A deficiência de G6PD é confirmada com a atividade enzimática abaixo de 12,5U/gmHb. Contudo, trata-se de um recurso demorado, trabalhoso e que requer um espectrofotômetro, não se mostrando adequado para testes em larga escala. Nesse sentido, recentemente propôs-se um novo teste como alternativa para uso em ambientes com poucos recursos, um biossensor eletroquímico que mede a transferência de elétrons da alteração do NADPH em sua forma reduzida usando as enzimas G6PD (PENGBOON P, et al., 2019; WENG Y, et al., 2003).

Sabendo-se que a deficiência de G6PD é uma doença genética dominante ligada ao $\mathrm{X}$ incompleta e que por isso é transmitida do pai para a filha e da mãe para metade de seus filhos, destaca-se como limitação para alcance do diagnóstico precoce o fato de que nem todas as mulheres heterozigotas serão identificadas com a doença por métodos bioquímicos. Além disso, vale ressaltar os altos custos necessários para testagem universal em locais de baixa incidência e a opinião, equivocada, compartilhada por muitos profissionais de que a enzimopatia é restrita a locais específicos (KAPLAN M e HAMMERMAN C, 2009; WATCHO JF, 2017; JIANG J, et al., 2014).

Dos estudos analisados, 50\% ressaltam a necessidade da triagem neonatal da deficiência de G6PD, sendo ela viável e econômica aos países, apresentando evidências de diminuição da incidência de hiperbilirrubinemia e kernicterus nos países que adotaram o programa (FARIA DC, et al., 2016; ISA HM, et al., 2017; OSHIRO M, et al., 2014; KAPLAN M e HAMMERMAN C, 2009).

\section{Prognóstico}

Nos neonatos deficientes de G6PD, é de suma importância que a alta hospitalar materna seja equiparada por informações necessárias para a detecção da icterícia. Alguns fatores para o desenvolvimento do kernicterus são observados, como a despreocupação por parte da equipe de saúde com os altos níveis da bilirrubina dos recém-nascidos durante a alta hospitalar, bem como possíveis fatores de risco para sua elevação.

Dessa maneira, neonatos ictéricos fora do ambiente hospitalar devem ser levados precocemente a uma assistência médica para avaliação da bilirrubina sérica total, sendo fundamental para diminuir os níveis dessa bilirrubina, caso se encontre elevada. Ressalta-se ainda que, se bebês prematuros forem tratados de forma parecida com os bebês a termo, com a não avaliação da bilirrubina na alta hospitalar, o risco de um pior prognóstico é mais elevado (FACCHINI FP, et al., 2017; KAPLAN M e HAMMERMAN C, 2009).

A hiperbilirrubnemia embora quase sempre benigna e reversível, mas se muito elevada, pode causar danos ao sistema nervoso e nesse contexto, o tratamento deve ser iniciado imediatamente para evitar que ocorra desfechos desfavoráveis, diminuindo assim a mortalidade, prevenindo as sequelas neurológicas graves e resultando em um melhor prognóstico no recém-nascido.

Porém, a falta de iniciativa diante do quadro de icterícia no neonato ou a não detecção precoce podem ser responsáveis pelo quadro de kernicterus, caracterizado por anormalidades funcionais que são observadas na maioria das crianças, como os distúrbios relacionados ao movimento, decorrente dessa impregnação de bilirrubina no sistema nervoso central. Concomitante a essas alterações, o quadro de kernicterus também pode levar ao desenvolvimento de outras alterações neurológicas, principalmente a nível de audição e visão (VINHAL RM, et al., 2009; SHAPIRO S, 2005).

Nos casos mais graves de kernicterus, os indivíduos podem parecer ter retardo mental grave, mas possuem inteligência normal ou superior, porém ficam presos em corpos imóveis e disfuncionais e não podem se mover, ouvir, assinar, digitar ou se comunicar de maneira voluntária (SHAPIRO S, 2005). A manifestação clínica da neurotoxicidade da bilirrubina varia com o local, a gravidade e o tempo da avaliação e é influenciada 
por fatores que incluem a quantidade, duração e idade de desenvolvimento da exposição à bilirubina livre excessiva. Portanto, o kernicterus devido à deficiência de G6PD é evitável desde que a icterícia seja diagnosticada precocemente e intervenções adequadas sejam prontamente instruídas (NAIR AK e KHUSAIBY SM, 2003; SHAPIRO S, 2005).

\section{Tratamento}

O manejo da deficiência de G6PD, consiste na prevenção a exposição ao estresse oxidativo e manejo das suas repercussões. No caso da hiperbilirrubinemia por G6PD, assim como naquelas de outras etiologias, o tratamento depende dos valores de bilirrubina sérica, podendo variar da conduta expectante, a fototerapia e a exsanguinotransfusão, sendo a última usada em casos de refratariedade ou hiperbilirrubinemias intensas (ISA HM, et al., 2017; KAPLAN M e HAMMERMAN C, 2002; KAPLAN M, et al., 2011).

A fototerapia é, sem dúvida, a terapêutica mais utilizada para o tratamento da icterícia. Seu sucesso depende da transformação fotoquímica da bilirrubina em produtos hidrossolúveis que serão excretados pelos rins ou pelo fígado. Ademais, sua eficácia também depende de fatores como a concentração inicial da bilirrubina antes do tratamento, a superfície corporal exposta à luz, a dose e a irradiância emitida e o tipo de luz utilizado.

Dois mecanismos têm sido propostos para explicar a ação da fototerapia na redução dos níveis séricos de bilirrubina: fotoisomerização e, em menor proporção, a fotooxidação. O primeiro e mais importante sistema baseia-se na propriedade da molécula de bilirrubina dar origem a dois tipos de isômeros: o isômero geométrico ou configuracional e o isômero estrutural ou lumirrubina. Sendo a formação desses isômeros estruturais ou lumirrubinas um processo irreversível e dada sua alta solubilidade em água, são rapidamente excretados pela bile e, principalmente, pela urina do recém-nascido ictérico em vigência do tratamento por fototerapia (CARVALHO M, 2001).

A exsanguinotransfusão, por sua vez, têm como intuito remover diretamente o excesso de bilirrubina circulante, prevenindo, desta maneira, seus efeitos tóxicos. Com esta técnica, cerca de $85 \%$ das hemácias na circulação são substituídas, promovendo uma queda na concentração sérica de bilirrubina de cerca de $50 \%$. Apesar de ser um procedimento relativamente seguro, possui complicações, como por exemplo tromboembolismo, enterite necrosante, perfuração vascular, hemorragias, distúrbios eletrolíticos, metabólicos, ácidos-básicos e a infecção, com taxas de mortalidade girando em torno de 1\%. Desse modo, as indicações de exsanguinotransfusão devem ser individualizadas, e o risco-benefício deve ser ponderado (CARVALHO M, 2001).

Existem ainda diversos outros recursos terapêuticos, entre eles o tratamento farmacológico. Entretanto, são meios ainda em constante evolução e estudo, com indicação pormenorizada a cada caso. Entre os mais pesquisados e colocados em prática, respectivamente, estão os inibidores da heme oxigenase e a gamaglobulina. Alguns estudos indicam ainda que neonatos com hiperbilirrubinemia apresentam níveis menores de vitaminas antioxidantes como $\mathrm{A}, \mathrm{C}$ e.

Porém, o tratamento com vitamina $E$ não se mostrou efetivo e, ainda que o uso de antioxidantes seja uma possibilidade de tratamento promissor, faltam estudos que abordem posologia, dose e momento adequado para sua reposição. Ainda, existem novas propostas de tratamento baseadas no desenvolvimento de moléculas ativadoras de G6PD, porém, essas ainda se mantém em constante testes e pesquisas (CARVALHO M, 2001; CUNNINGHAN AD, et al., 2016; KAPLAN M e HAMMERMAN C, 2004).

\section{CONSIDERAÇÕES FINAIS}

Conclui-se que neonatos com déficit da enzima G6PD são significativamente mais propensos a desenvolver quadros de hiperbilirrubinemia, podendo esta condição, quando patológica, evoluir para encefalopatia bilirrubínica crônica $(E B C)$ ou Kernicterus, progredindo para paralisia cerebral do tipo atetóide ou até mesmo óbito nos quadros mais graves. A prevenção destas complicações baseia-se no manejo da hiperbillirubinemia, principalmente por meio da fototerapia e, em casos refratários, pela exsanguinotransfusão. A busca por um bom prognóstico envolve diretamente a precocidade do diagnóstico e do tratamento. Sendo assim, é fundamental a conscientização dos pais e responsáveis quanto à necessidade de manterem-se vigilantes à possíveis alterações e serem ágeis na procura pela assistência médica. 


\section{REFERÊNCIAS}

1. BRASIL. Ministério da saúde. Triagem neonatal para deficiência de enzima desidrogenase de glicose hepática (glicose-6- fosfato desidrogenase, G-6-PD). Brasília, 2018.

2. CARVALHO M. Tratamento da icterícia neonatal. Jornal de Pediatria, 2001; 77 (1): 71-80.

3. CUNNINGHAM AD, et al. Glucose-6-Phosphate Dehydrogenase Deficiency and the Need for a Novel Treatment to Prevent Kernicterus. Clinics in Perinatology, 2016; 43(2): 341 - 354.

4. FACCHINI FP, et al. Follow-up of neonatal jaundice in term and late premature newborns. J Pediatr, 2017; 83(4): 313-318.

5. FARIA DC, et al. Manifestações clínicas em crianças portadoras da deficiência de glicose-6-fostato desidrogenase (G6PD): revisão integrativa. Revista de Medicina e Saúde de Brasília, 2016; 5(2).

6. FRANK JE. Diagnosis and Management of G6PD Deficiency. American Family Physician, 2005; 72(7): $1277-1282$.

7. IGLESSIAS MAC, et al. Erythrocyte glucose-6-phosphate dehydrogenase deficiency in male newborn babies and its relationship with neonatal jaundice. Revista Brasileira de Hematologia e Hemoterapia, 2010; 32(6): 434-438.

8. ISA HM, et al. Neonatal indirect hyperbilirubinemia and glucose-6-phosphate dehydrogenase deficiency. Clin Exp Pediatr., 2017; 60(4): 106-111.

9. JIANG J, et al. Screening and prevention of neonatal glucose 6-phosphate dehydrogenase deficiency in Guangzhou, China. Genetics and Molecular Research, [s.I.]; 13(2): 4272-4279.

10. KAPLAN M, et al. Severe Neonatal Hyperbilirubinemia and Kernicterus: Are These Still Problems in the Third Millennium? Neonatology, 2011; 100: 354-362.

11. KAPLAN M, HAMMERMAN C. Glucose-6-phosphate dehydrogenase deficiency: a potential source of severe neonatal hyperbilirubinemia and kernicterus. Semin Neonatol, 2002; 7:121-128.

12. KAPLAN M, HAMMERMAN C. Glucose-6-phosphate dehydrogenase deficiency: a hidden risk for kernicterus. Seminars in Perinatology, 2004; 28(5):356-364.

13. KAPLAN M, HAMMERMAN C. The need for neonatal glucose-6-phosphate dehydrogenase screening: a global perspective. Journal of Perinatology, 2009; 29: S46-S52.

14. LEITE AA. Icterícia neonatal e deficiência de glicose-6-fosfato desidrogenase. Rev Bras Hematol Hemoter., 2010; 32(6):430-431.

15. NAIR AK, KHUSAIBY SM. Kernicterus and G6PD Deficiency-a Case Series from Oman. Journal Of Tropical Pediatrics, 2003; 49(2): 74-77.

16. NKHOMA ET, et al. The global prevalence of glucose-6-phosphate dehydrogenase deficiency: a systematic review and meta-analysis. Blood Cells Mol Dis, 2009; 42(3): 267-278.

17. OSHIRO M, et al. Deficiência de glicose-6-fosfato desidrogenase na icterícia neonatal: avaliação de 10 anos do Instituto Adolfo Lutz-SP. Boletim do Instituto Adolfo Lutz, 2014; 1(24): 33-35.

18. PENGBOON $P$, et al. Evaluation of quantitative biosensor for glucose-6-phosphate dehydrogenase activity detection. Plos One, 2019; 14(12):1-14.

19. RADMACHER PG, et al. A modified Bilirubin-induced neurologic dysfunction (BIND-M) algorithm is useful in evaluating severity of jaundice in a resource-limited setting. Bmc Pediatrics, 2015; 15(1).

20. SHAPIRO S. Definition of the Clinical Spectrum of Kernicterus and Bilirubin-Induced Neurologic Dysfunction (BIND). J Perinatol, 2005; 25: 54-59.

21. USMAN F, et al. Acute bilirubin encephalopathy and its progression to kernicterus. Reserach and Reports In Neonatology, 2018; 8(1): 33-44.

22. VINHAL RM. Icterícia neonatal e Kernicterus: conhecer para prevenir. Revista Movimenta, 2009; 2(3).

23. WATCHKO JF. Refractory Causes of Kernicterus in Developed Countries: Can We Eradicate G6PD Deficiency Triggered and Low-Bilirubin Kernicterus? Current Pediatric Reviews, 2017; 13:159-168.

24. WENG Y, et al. Hyperbilirubinemia in healthy neonates with glucose-6-phosphate dehydrogenase deficiency. Early Human Development, 2003; 71(2): 129-136.

25. WHO WG. Glucose-6-phosphate dehydrogenase deficiency. Bull World Health Organ, 1989; 67:601-11. 